Proceedings

\title{
Autocrine Growth Hormone Mediated Curcumin Resistance Overcame by Autophagy Inhibition via Bafilomycin in MDA-MB-231 and T47D Breast Cancer Cells ${ }^{\dagger}$
}

\author{
Derya Bulut *, Ajda Coker-Gurkan, Recep Genc, Elif Damla Arisan, Pınar Obakan-Yerlikaya and \\ Narcin Palavan-Unsal \\ Department of Molecular Biology and Genetics, Faculty of Science and Letters, Istanbul Kultur University, \\ Istanbul 34156, Turkey; a.coker@iku.edu.tr (A.C.-G.); recogenc94@gmail.com (R.G.); \\ d.arisan@iku.edu.tr (E.D.A.); p.obakan@iku.edu.tr (P.O.-Y.); n.palavanunsal@iku.edu.tr (N.P.-U.) \\ * Correspondence: buluttderya@gmail.com \\ + Presented at the 2nd International Cell Death Research Congress, Izmir, Turkey, 1-4 November 2018. \\ Published: 10 December 2018
}

\begin{abstract}
Curcumin, a plant derived natural compound, has anti-oxidant, anti-proliferative and apoptotic effect on various cancer cells such as prostate, colon and breast cancer. Autocrine growth hormone $(\mathrm{GH})$ expression induced breast cancer invasion-metastasis has been reported in vivo and in vitro cancer models. Autophagy is a vesicule-mediated clearance mechanism and one of the handicap against drug-induced apoptotic cell death. In this study, our aim was to investigate the molecular machinery of curcumin induced apoptotic cell death under autophagy inhibition conditions in autocrine GH expressing MDA-MB-231 and T47D breast cancer cells. Although autocrine $\mathrm{GH}$ induced curcumin resistance, this effect was slightly prevented by time-dependent curcumin treatment in MDA-MB-231 and T47D breast cancer cells. In addition, curcumin induced autophagy vacuole formation was determined by acridine orange staining in MDA-MB-231 and T47D wt/GH+ breast cancer cells. Moreover, curcumin triggered autophagy through upregulating Beclin-1, Atg3, Atg12 expressions and LC3 cleavage in each cell line. Concomitantly, BiP, IRE1 $\alpha$ and Calreticulin expressions were upregulated following $3 \mathrm{~h}$ curcumin exposure in MDA-MB-231 wt and GH+ cells. According to MTT cell viability assay, autocrine GH-mediated curcumin resistance was overcome by bafilomycin and curcumin co-treatment in MDA-MB-231 and T47D GH+ cells. Moreover, curcumin and bafilomycin co-treatment induced cell cycle arrest at G1 phase in MDAMB-231 GH+ cells, G2/M arrest in T47D GH+ breast cancer cells. In conclusion, autocrine GHtriggered curcumin resistance was overcome by autophagy inhibition condition by bafilomycin treatment in a dose-dependent manner in MDA-MB-231 and T47D GH+ breast cancer cells.
\end{abstract}

Keywords: curcumin; bafilomycin; autophagy; breast cancer; growth hormone

\section{Introduction}

Breast cancer is one of the most common cancers among women, and fifth causes of cancerrelated death in worldwide [1]. Growth hormone $(\mathrm{GH})$ is a protein-derived hormone secreted fundamentally from the anterior pituitary gland and performs crucial tasks in mammary gland development during puberty [2,3]. In addition, both GH and its receptor (GHR) expression profiles were upregulated in various cancers such as prostate, colon and breast cancer [4,5]. Curcumin, is a plant derived compound extracted from Curcuma longa, has anti-inflammatory, anti-oxidant, antiproliferative and anti-carcinogenic effects [6]. Dose- and time-dependent curcumin exposure induced 
apoptotic cell death via acting on endoplasmic reticulum (ER) stress in cancer cells [7]. In addition, autophagy, a cellular process under nutrient deprivation, growth factor depletion, and hypoxia conditions, is activated during curcumin triggered apoptotic cell death in colon, cervical and breast cancers [8].

Our aim, in this study is to demonstrate the role of autophagy and ER stress in curcumin induced apoptotic cell death in MDA-MB-231 (ER-) and T47D (ER+) breast cancer cells and also modulate the apoptotic potential of curcumin under autophagy suppression condition by bafilomycin.

\section{Material and Methods}

Time-dependent curcumin (Sigma Aldrich, C1386, Munich, Germany) effect on cell growth and proliferation was determined by Mitotracker/DAPI (Thermo fisher Scientific, Mitotracker Red CMXRos, M7512, DAPI, D1306, Waltham, MA, USA) staining in T47D (ATCC, HTB-133, Manassas, VA, USA) and MDA-MB-231 (ATCC, HTB-26, Manassas, VA, USA) breast cancer cells. The potential additional effect of bafilomycin (Abcam, ab120497, Cambridge, UK) on curcumin induced cell viability loss was determined by MTT (Sigma Aldrich, M2128, St. Louis, MO, USA) cell viability assay. Bafilomycin and curcumin co-treatment on cell cycle and apoptotic cell death was demonstrated by PI and Annexin V/PI analysis, respectively (BD Biosciences, 556547, San Jose, CA, USA). Time-dependent curcumin effect on autophagy (CST, Autophagy antibody sampler kit, 4445, Danvers, MA, USA) and ER stress (CST, ER stress antibody sampler kit, 9956, Danvers, MA, USA) was determined by immunoblotting.

\section{Results and Discussion}

Autocrine GH induced cell growth and proliferation in MDA-MB-231 and T47D breast cancer cells. However, this effect was diminished by dose- and time-dependent curcumin treatment in each breast cancer cells (Figure 1a,b). Concomitantly, curcumin triggered Beclin-1, Atg3, Atg12 gene expression upregulation and LC3 cleavage in MDA-MB-231 GH+ cells. Moreover, time-dependent curcumin exposure induced Calreticulin and IRE1 $\alpha$ expressions in each forced GH expressing breast cancer cells (Figure 2). According to the MTT cell viability results, curcumin induced cell viability loss increased from $41.5 \%$ to $54.24 \%$ by bafilomycin co-treatment in MDA-MB-231 GH+ cells. Similarly, in T47D GH+ cells, $20 \mu \mathrm{M}$ curcumin for $24 \mathrm{~h}$ triggered $29.68 \%$ cell viability loss was accelerated to $35.94 \%$ by bafilomycin treatment in $\mathrm{T} 47 \mathrm{GH}+$ breast cancer cells (Figure 3a,b). According to PI analysis, curcumin and bafilomycin co-treatment induced cell cycle arrest at G1 phase in MDA-MB-231 and G2/M phase in T47D breast cancer cells. According to Annexin V/PI staining, apoptotic cell population was $1.5 \%$ and $8.7 \%$ by curcumin treatment in MDA-MB-231 GH+ and T47D GH+ breast cancer cells, respectively. However, curcumin induced apoptotic population was increased by bafilomycin and curcumin co-treatment (3.2\% in MDA-MB-231 GH+, 11.8\% in T47D $\mathrm{GH}+)$ (Data not shown).

Autocrine GH signaling has been assumed to be an obstacle for the therapeutic effect of drugs by triggering cancer drug resistance profile and new strategies should be taken to overcome autocrine GH mediated drug resistance in breast cancer cells. Thus, autocrine GH mediated curcumin resistance might be overcome by bafilomycin treatment in order to prevent drug induced autophagy regulation in MDA-MB-231 and T47D breast cancer cells. 

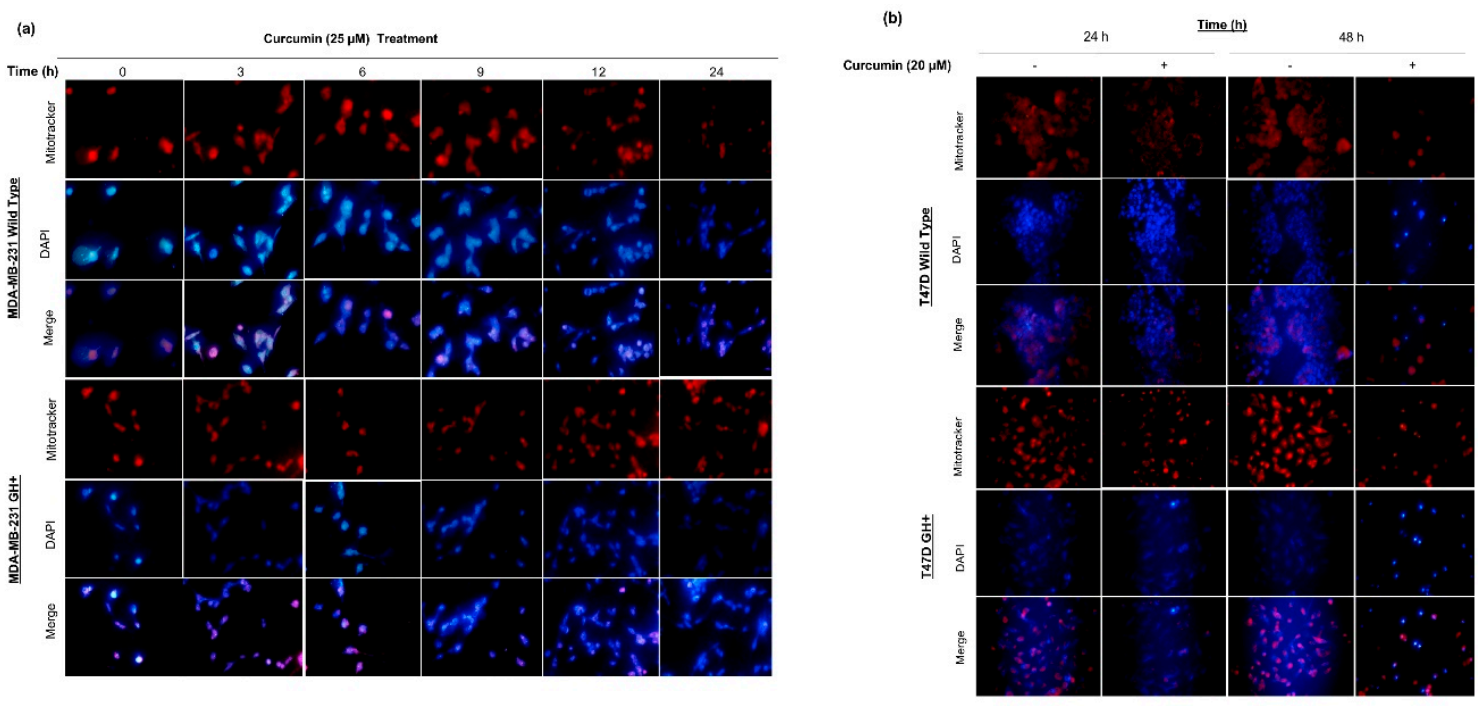

Figure 1. Curcumin induces cell viability loss in time dependent manner. (a) MDA-MB-231 wt and $\mathrm{GH}+$; (b) T47D wt and GH+ breast cancer cells were seeded in 6 well-plate and curcumin ( $25 \mu \mathrm{M}$ for MDA-MB-231 and $20 \mu \mathrm{M}$ for T47D cells) exposed to cells in time-dependent $(0-24 \mathrm{~h})$ manner. Following drug exposure cells were stained by Mitotracker for $15 \mathrm{~min}$ and DAPI staining for $5 \mathrm{~min}$, respectively and Mitotracker/DAPI stained cells were visualized by fluorescence microscopy.

MDA-MB-231 Wild Type

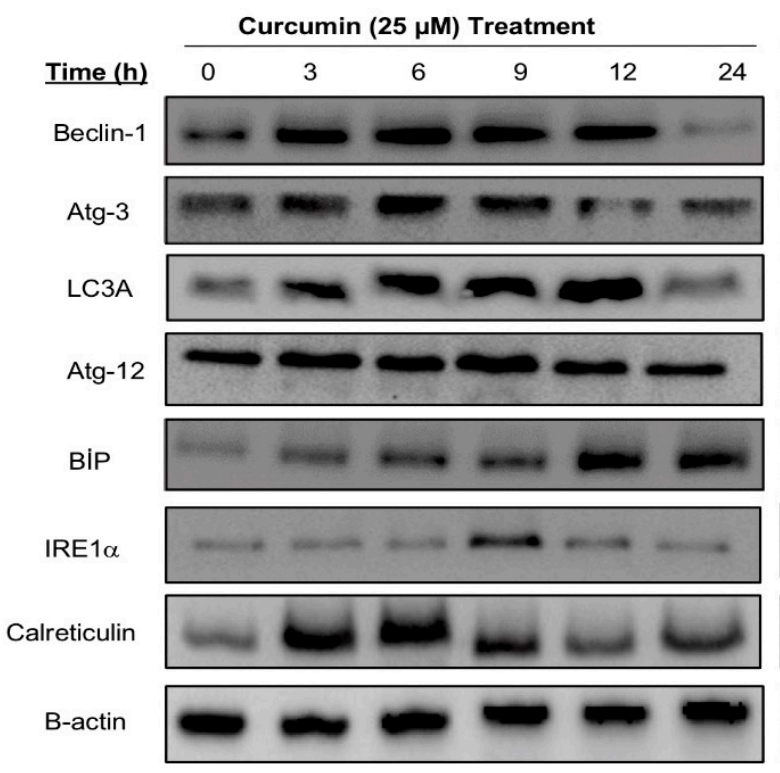

MDA-MB-231 GH+

\section{Curcumin $(25 \mu \mathrm{M})$ Treatment}

\begin{tabular}{llllll}
\hline 0 & 3 & 6 & 9 & 12 & 24
\end{tabular}
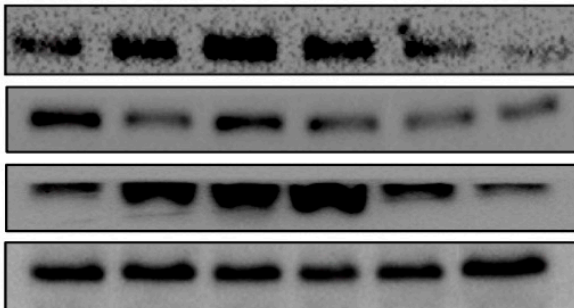

$60 \mathrm{kDa}$

$40 \mathrm{kDa}$

14-16 kDa

$55,16 \mathrm{kDa}$

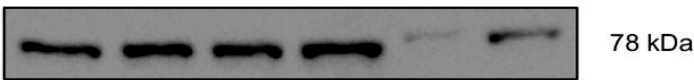

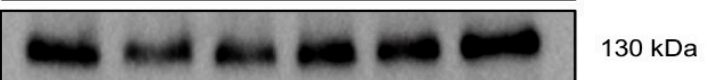
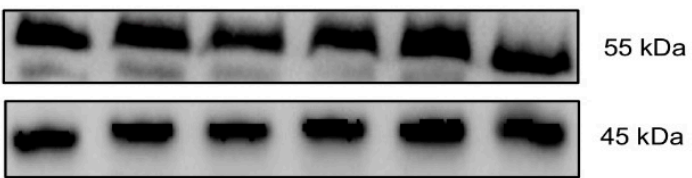

Figure 2. Time-dependent curcumin induced autophagy and ER stress in MDA-MB-231 and T47D $\mathrm{wt} / \mathrm{GH}+$ breast cancer cells. The time dependent effect of curcumin on autophagy key players (Beclin1, Atg3, Atg12, LC3) and ER stress (BiP, IRE1 $\alpha$ and Calreticulin) molecules expression profile was determined by immunoblotting. 


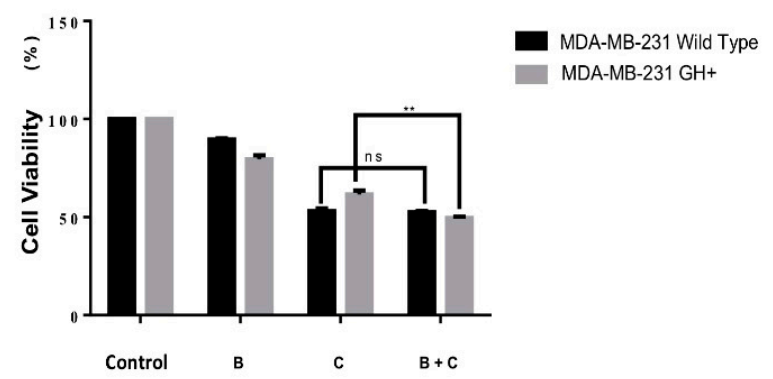

(a)

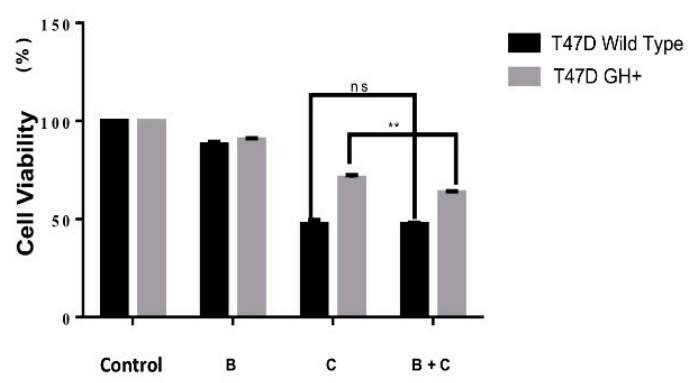

(b)

Figure 3. Effect of bafilomycin on curcumin-induced cell viability loss. Determined dose of curcumin (25 $\mu \mathrm{M}$ for MDA-MB-231 and $20 \mu \mathrm{M}$ for T47D cells) for $24 \mathrm{~h}$ treatment with/without Bafilomycin (50 $\mathrm{nM}$ ) in (a) MDA-MB-231 wild type and GH+ and (b) T47D wild type and GH+ and MTT cell viability assay was performed. The columns represent mean \pm S.D. of two independent experiments and at least 3 replicates. B: Bafilomycin, C: Curcumin, B + C: Bafilomycin and Curcumin.

\section{Conclusions}

Autocrine GH expression induced cell proliferation, growth and invasion-metastatic profile and also curcumin resistance in MDA-MB-231 and T47D breast cancer cells. However, forced GH mediated curcumin resistance overcome by time-dependent drug exposure. As curcumin induced fluctuation in autophagy and ER stress key markers expression profile in MDA-MB-231 and T47D breast cancer cells, curcumin triggered cell viability loss and apoptotic cell death was accelerated under autophagy suppression condition by bafilomycin treatment in each breast cancer cells.

\section{References}

1. Subramani, R.; Nandy, S.B.; Pedroza, D.A.; Lakshmanaswamy, R. Role of Growth Hormone in Breast Cancer. Endocrinology 2017, 158, 1543-1555.

2. Emerald, B.S.; Chen, Y.; Zhu, T.; Lee, K.O.; Gluckman, P.D.; Lobie, P.E. $\alpha$ CP1 mediates stabilization of hTERT mRNA by autocrine human growth hormone. J. Biol. Chem. 2007, 282, 680-690.

3. Zhu, T.; Goh, E.L.; Graichen, R.; Ling, R.; Lobie, P.E. Signal transduction via the growth hormone receptor. Cell Signal. 2001, 13, 599-616.

4. Perry, J.K.; Emerald, B.S.; Mertani, H.C.; Lobie, P.E. The oncogenic potential of growth hormone. Growth Horm. IGF Res. 2006, 16, 277-89.

5. Conway-Campbell, B.L.; Wooh, J.W.; Brooks, A.J.; Gordon, D.; Brown, R.J.; Lichanska, A.M.; Chin, H.S.; Barton, C.L.; Boyle, G.M.; Parsons, P.G.; et al. Nuclear targeting of the growth hormone receptor results in dysregulation of cell proliferation and tumorigenesis. Proc. Natl. Acad. Sci. USA 2007, 104, 13331-13336.

6. Wilken, R.; Veena, M.S.; Wang, M.B.; Srivatsan, E.S. Curcumin: A review of anti-cancer properties and therapeutic activity in head and neck squamous cell carcinoma. Mol. Cancer 2011, 10, 12.

7. Zhang, L.; Cheng, X.; Xu, S.; Bao, J.; Yu, H. Curcumin induces endoplasmic reticulum stress-associated apoptosis in human papillary thyroid carcinoma BCPAP cells via disruption of intracellular calcium homeostasis. Medicine 2018, 97, e11095.

8. Levine, B.; Kroemer, G. Autophagy in the pathogenesis of disease. Cell 2008, 132, 27-42.

(C) 2018 by the authors. Licensee MDPI, Basel, Switzerland. This article is an open access article distributed under the terms and conditions of the Creative Commons Attribution (CC BY) license (http://creativecommons.org/licenses/by/4.0/). 